\title{
Analysis of the insertion of solar Photovoltaic generation in large consumers of Rio de Janeiro: A Case Study
}

\author{
Nogueira, P.C. ${ }^{1}$, Sousa, C.R. ${ }^{2}$ \\ ${ }^{1}$ Department of Civil Engineering \\ ${ }^{2}$ Department of Electrical Engineering \\ PUC-Rio, Pontifical Catholic University of Rio de Janeiro \\ Marquês de São Vicente, 225. Gávea. Rio de Janeiro, RJ. (Brazil) \\ E-mail: pedrocomarella@gmail.com, reinaldo@ele.puc-rio.br
}

\begin{abstract}
The last few years have shown an exponential growth in the use of photovoltaics in the world. The worldwide installed capacity of $23 \mathrm{GW}$ in 2009 reached the expressive mark of $139 \mathrm{GW}$ in 2013, being the European continent the holder of the largest portion of it $(59 \%$ or $81 \mathrm{GW})$. The most recent estimates describe a still small market in Brazil, with only 20 MW of installed capacity (EPE 2014) and mostly located in remote areas not covered by the interconnected system. Nevertheless, the growth potential of the domestic market is promising, greater even than that presented by the leaders in the use of this source. In this paper it is presented an implementation of a solar photovoltaic project in one medium voltage client of the distributing utility of Rio de Janeiro (LIGHT). The choice of this client was based in a previous work by (Carmo, 2014 \& 2015) that used a fuzzy logic system to classify the potential medium and high voltage clients of LIGHT based on the matching of their weekdays load shape curves and the corresponding daily solar radiation curve in Rio de Janeiro. The results show that, depending on the values of key variables (tariff, rate of exchange and price of equipment) the project is economically viable.
\end{abstract}

\section{Key words}

Photovoltaic Solar Energy, Economic \& Financial Feasibility, Medium \& High Voltage Clients, LIGHT Distributing Utility

\section{Introduction}

The last few years have shown an exponential growth in the use of photovoltaics in the world. The worldwide installed capacity of $23 \mathrm{GW}$ in 2009 reached the expressive mark of $139 \mathrm{GW}$ in 2013, being the European continent the holder of the largest portion of it $(59 \%$ or 81GW). (EPIA, 2011 \& 2013).

The most recent estimates describe a still small market in Brazil, with only $20 \mathrm{MW}$ of installed capacity (EPE 2012 \& 2014) and mostly located in remote areas not covered by the interconnected system. Nevertheless, the growth potential of the domestic market is promising, greater even than that presented by the leaders in the use of this source.

Based on the inherent natural advantages that the country possesses (such as high levels of solar radiation), it's safe to say that the photovoltaic power generation can be an important driver to increase the participation of renewable sources and the security of our energy matrix.

In fact, this movement was already anticipated by planners of the electric sector. The power Expansion Plan with 2024 horizon PDE 2024, prepared by the Energy Research Company (EPE), was published in December 2015. Among the main projections of the plan is the increase of the installed capacity of power generation in 73.000 MW. Half of this expansion is based on renewable sources (wind, solar, biomass and small hydropower plants), and 7.000 MW exclusively from photovoltaic generation.

In this context, this paper seeks to contribute to the deepening of the studies devoted to the nascent national photovoltaic market, covering in particular the Estate of Rio de Janeiro.

We sought to evaluate the economic feasibility of photovoltaic distributed generation systems for large customers of the concessionaire Light, estimating not only the price of a photovoltaic system of medium/large size but also the financial return and sensitivity analysis contemplating multiple scenarios.

Subsequently, the economic feasibility analysis developed was applied to a real client of Light in Rio de Janeiro, chosen among the scope of consumers holding high technical potential for photovoltaic use.

Undeniably, the current scenario of the Brazilian electric sector, with lower levels in the reservoirs and continued use of thermal power plants in order to meet the demand, 
indicates a substantial increase in viability conditions of adopting a photovoltaic alternative.

The consumer decision of adopting solar generation in a establishment depends directly on the comparative analysis of how much he wouldn't be paying the distributor for purchasing energy (since he would produce the energy himself) versus the amount of investment necessary to acquire and maintain the photovoltaic system.

Therefore, an economic and financial evaluation of photovoltaic systems was made having as interest group commercial/industrial consumers belonging to Light's A group (connected to medium and high voltage).

\section{Methodology}

In order to implement the project, we used the usual tools of financial mathematics (NPV-net present value, internal rate of return-IRR and payback) to perform a feasibility analysis of investment in the photovoltaic system, taking into consideration two generic consumer profiles.

Aligned to the exercise directed by EPE (2012), we assumed a $100 \mathrm{kWp}$ system as being representative of a typical commercial installation and a $1000 \mathrm{kWp}$ system as being representative of a typical industrial facility.

The analysis is based on a cash flow projection comprising the entire life of the system (usually 25 years), in which are considered all revenue entries (cost avoided by not buying energy from distributor) and outflows brought to present value in order to be compared to the initial investment value.

It was also considered that the total cost of the photovoltaic system is composed of modules, inverter, BOS (Balance of System Costs) and the costs of installation. The BOS in turn includes the electrical components, dimensioning, installation and support of the system, cables, etc.

In order to determine the final cost in terms of $\mathrm{R} \$ / \mathrm{W}$ (Reais per installed Watt) it was used the recommendations proposed by ABINEE (2012) in the study "Proposals for the insertion of solar photovoltaic energy within the Brazilian electrical energy matrix”.

The estimated price was $\mathrm{R} \$ 5.64 / \mathrm{Wp}$. This number is consistent with the original value estimated by ABINEE (2012) for a similar system, between $\mathrm{R} \$ 6.27 / \mathrm{W}$ and $\mathrm{R} \$ 5.37 / \mathrm{W}$.

\section{Scenarios description}

In addition to the price of the system, other variables were considered in order to draw up the multiple future scenarios that comprised the analysis. The default scenario may be found below:

\begin{tabular}{|l|l|}
\hline Parameters & Value \\
\hline Useful life of system & 25 years \\
\hline Exchange rate $\mathrm{R} \$ / € \$$ & 3,30 \\
\hline System price $\mathrm{R} \$ \mathrm{Wp}$ & 5,64 \\
\hline O\&M cost & $1,00 \%$ per year \\
\hline PR (performance rate) & $75 \%$ \\
\hline d (annual module degradation) & $0,50 \%$ per year \\
\hline FC (capacity factor) & $15,10 \%$ \\
\hline A4 group electric rate & $\begin{array}{l}625.81 \\
\mathrm{R} \$ \mathbf{M W h}\end{array}$ \\
\hline Inflation & $5,56 \%$ per year \\
\hline $\begin{array}{l}\text { minimum attractive rate of } \\
\text { return }\end{array}$ & $\begin{array}{l}13,75 \% \\
\text { year }\end{array}$ \\
\hline
\end{tabular}

In addition to the default scenario formalized above, other 11 scenarios were calculated in order to test various impacts on the input variables.

It was used different combinations of inflation rates (determinants of electric rate increase), discount rates (or minimum attractive rate of return) and financing of the initial investment options.

\section{Simulation results}

The results of calculations performed for the default scenario and for the 11 alternative scenarios considering the different system sizes can be seen below:
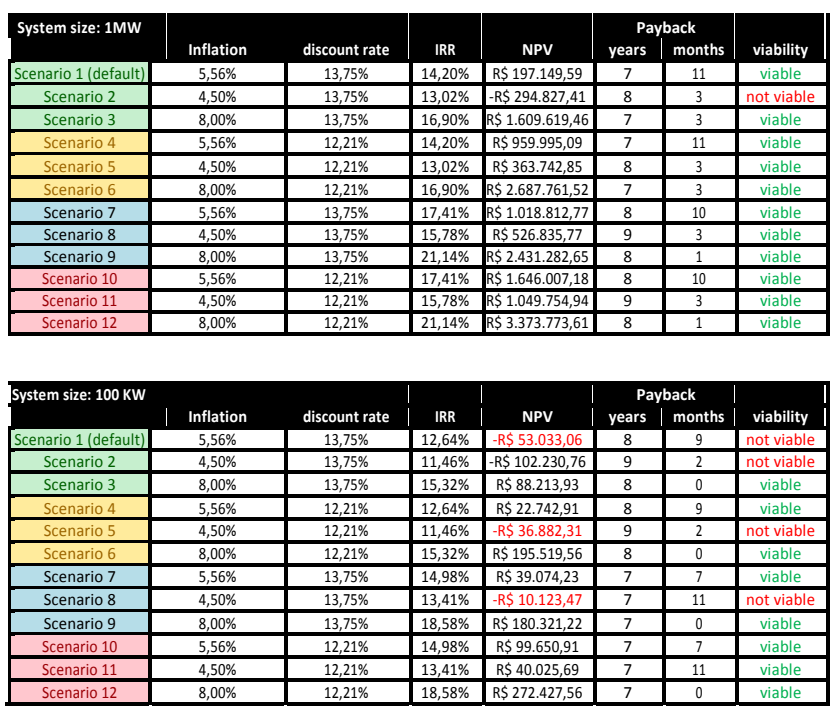

Considering the average exchange rate found in the first half of 2015 (3,30R\$/€), a majority condition of feasibility was observed among the 12 proposed scenarios.

The larger system only showed as not viable under the most "aggressive" proposed scenario, while the smaller one showed viability in 8 of them.

Then, a big client of the concessionaire Light in Rio de Janeiro was chosen among the scope of customers 
categorized as holders of high technical potential by CARMO (2014). In his study, CARMO (2014) used actual data provided by Light to build load curves of 4.767 customers, which after being compared with the curve of solar radiation incidence in Rio de Janeiro allowed the proper identification and classification of technically qualified customers to adopt the photovoltaic generation in their establishments.

The chosen client, Palace of th Finance Ministery, is one of the most iconic buildings in the city and one of the greatest representatives of the neo-classical architectural style in national territory. For details, see the Appendix.

Using Sketchup Pro 2015 software, a 3D model of the building was made and the path taken by the shadows of its own constructive elements as well as of other relevant buildings in its surroundings were simulated.

Thus, it was possible to delimit the roof area in which relevant shading occurs throughout the year, so as to derail the installation of photovoltaic plates in this perimeter.

To quantify the photovoltaic generation, different module orientation/tilt proposals were evaluated, being chosen the one that presented better results during summer months, in order to reconcile increased levels of radiation with the maximization of useful available surface usage, without neglecting the aesthetic factor.

A potential $583.18 \mathrm{KW}$ system was designed, being capable of generating an estimated value of 715.5 MWh per year. The power generated would be able to provide $11.68 \%$ of the annual need of the building, being the annual savings of approximately R $\$ 447.767,39$.

Once the power generation was estimated, it became possible to apply the previously developed feasibility study to the Palacio da Fazenda case study.

\section{Discussion and final remarks}

Projecting the future generation of energy for the entire useful life of the system (25 years) and using the same parameters estimated before, it was possible to analyze the financial feasibility of the project in each of the 12 chosen scenarios.

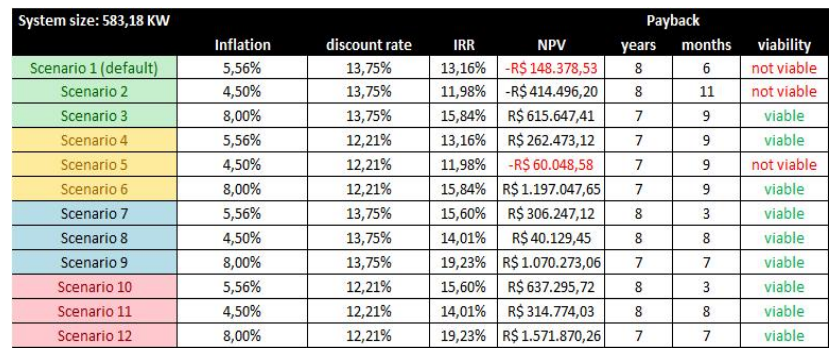

Most of the scenarios presented feasibility (9 of 12 showed positive net present values).
Two additional currency pricing scenarios were examined, both of them more disadvantageous from the standpoint of a module importer (4.00 $\mathrm{R} \$ / €$ and 4.50 $\mathrm{R} \$(€)$.

Clearly, the exchange rate indeed represents a critical factor in the analysis, since four additional scenarios became not viable using $4.00 \mathrm{R} \$ / €$ rate and only two of them remained viable under $4.50 \mathrm{R} \$$ /€ rate (only those which required a lower minimum attractiveness of $12.21 \%$, and had an above-inflation tariff precification remained viable).

However, it is important to realize that the evolution of electric rate above inflation was indeed repeatedly verified in the recent history of the country, being this a pattern that most likely will keep happening in near future.

\section{Appendix}

In this appendix it is displayed some of the important details of selected building to develop the solar energy project. It is a 14 floor building that is used as the Palace of the Ministry of Finance, located at the heart of Rio de Janeiro downtown. Figure A1 shows the average profile load curves of the high potential for implementing a photovoltaic system due to their coincidence of their high consumption (in blue) with high solar radiation (in red).

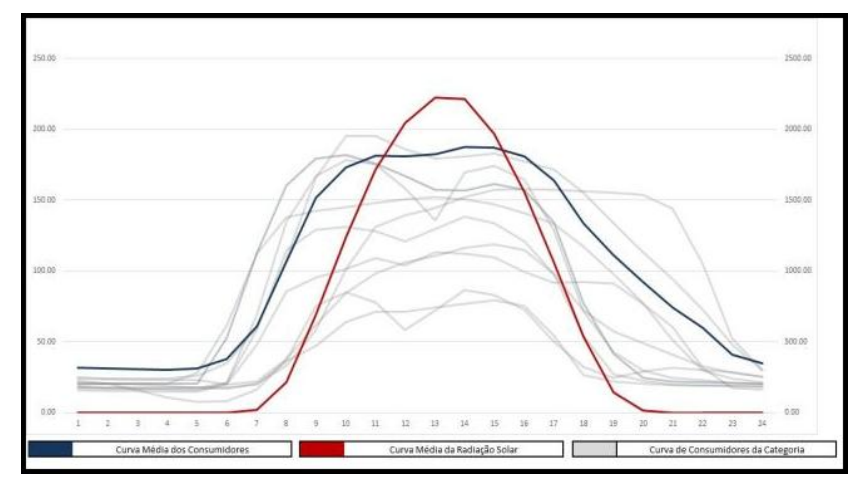

Figure A1: Average curve of solar radiation in Rio de Janeiro and average load shape curve from the high potential Light clients

Source: Carmo, (2014)

Figure A2 display a picture of the Palace which is located at the very heart of Rio de Janeiro city centre. As one can see, it is surrounded by other building (all the ministries buildings are located in the same avenue). Fortunately they have all the same structure and height, which reduces possible shadows in the roof of the building.

Finally, figure A3 display the working day load shape electricity consumption of the Palace and the corresponding solar radiation curve (year average) of Rio de Janeiro city. From thess curves, one can clearly see that, although it is a vertical building with a limited roof area the coincidence of the peak consumption with peak radiation implies that the project may be viable. 


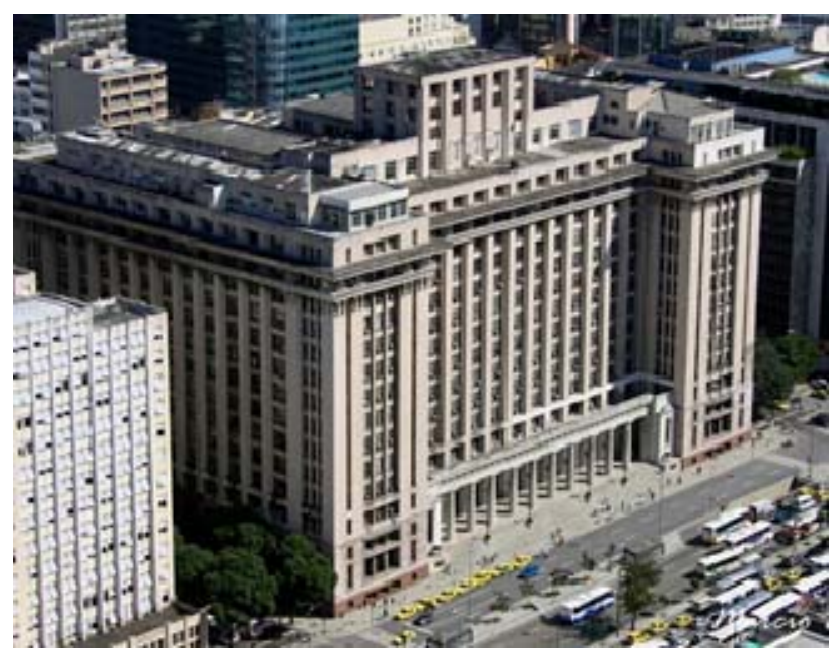

Figure A2: Selected buiding for the experiment (Labour Palace) Source: Nogueira, (2016)

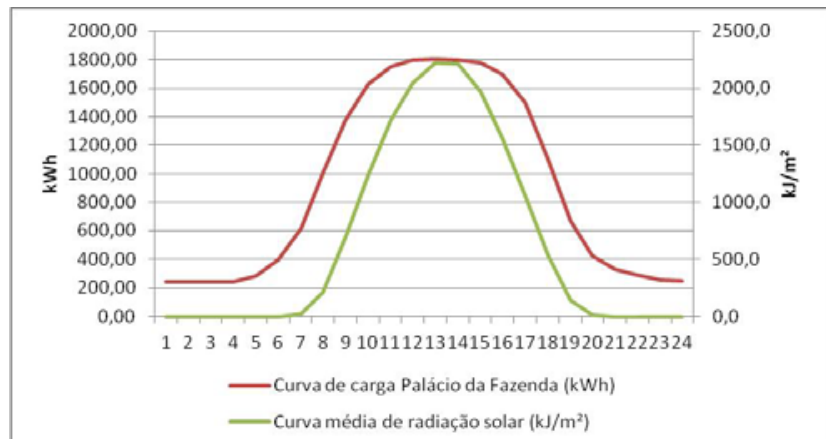

Figure A3: Palace load shape profile (working day) x solar radiation curve of Rio de Janeiro

Source: Nogueira (2016)

\section{References}

[1] ABINEE (2015): Proposals of Insertion of Solar Photovoltaic Energy within the Brazilian Electrical Matrix. In www.abinee.org.br (in Portuguese)

[2] CARMO, D.L. (2014): A Method to Identify potential Photovoltaic clients in a Large Distributing Utility of Brazil. Master Thesis, Pos MQI, PUC-Rio, Brazil (In Portuguese)

[3] CARMO, D.L. (2015): Identification of Potential Users of Photovoltaic Conversion. Annals of ICREPQ 2015, La Coruña, Spain

[4] EPE (2012): Analysis of the Insertion of the Solar Power within the Brazilian Electrical Generation System. In: www.epe.gov.br (In Portuguese)

[5] EPE (2014): An overview of the Electrical Generation in Brazil till 2024. In: www.epe.gov.br (In Portuguese)

[6] EPIA (2011): Solar Photovoltaics Competing in the Energy Sector - On road for Competitiveness. EPIA Report. In: www.epia.org

[7] EPIA (2013): Global Market Outlook. EPIA Report. In: www.epia.org
[8] NOGUEIRA, P. C. (2016): Analysis of the insertion of photovoltaic generation in medium/high voltage clients of Rio deJaneiro: A case study. Master thesis, Urban \& Environmental Post Doctorate program, PUCRio (In Portuguese) 